\title{
Leibniz: Regressus, un cálculo para la Silogística
}

\section{Leibniz: Regressus, a calculus for Syllogistic}

\author{
ANTONIO BENÍTEZ \\ JOSÉ MARÍA BENÍTEZ ESCARIO \\ Universidad Complutense de Madrid
}

Recibido: 31-10-2007

Aprobado definitivamente: 21-03-2009

\section{RESUMEN}

En este trabajo presentamos el cálculo para la Silogística que Leibniz llamó «Regressus». En la sección primera exponemos desde la notación usada por el propio Leibniz hasta el método de producción de los modos no perfectos. En la sección segunda comparamos la «fundamentación» de la Silogística de Leibniz con la que dio Aristóteles. En la sección tercera consideramos la hipótesis de Couturat según la cual se trata de un método cuya única estrategia demostrativa es la reducción al absurdo. En la sección cuarta lo presentamos como un algoritmo de producción de los modos imperfectos de la segunda y tercera figuras. Y concluimos, sección quinta, intentando hacer ver que, si se da «la vuelta» a dicho algoritmo, se obtiene un método de decisión para la Silogística con tres figuras.

\section{PALABRAS CLAVE}

LEIBNIZ, SILOGÍSTICA, HISTORIA DE LA LÓGICA

\begin{abstract}
In this paper we present the calculus for Syllogistic that Leibniz named «Regressus». In the first section we will show the notation used by Leibniz and the method of production of the imperfect moods. In the second section we will compare the ground of Leibniz's Syllogistic with Aristotle's. In the third section we will explore Couturat's hypothesis that holds that it is a method whose only demonstrative strategy is a reductio ad absurdum. In the fourth section we
\end{abstract}


will present it as a production algorithm of the imperfect moods of second and third figures. And we will end, in the fifth section, trying to show that, if we «turn over» that algorithm, we obtain a method of decision for Syllogistic of three figures.

KEYWORDS

LEIBNIZ; SYLLOGISTIC; HISTORY OF LOGIC

\section{El CÁlCULO DEL REGRESSUS}

Este cálculo se encuentra en el breve escrito «De formis syllogismorum Mathematice definiendis» de Leibniz. ${ }^{1}$

\section{I.1. LA NOTACIÓN}

- Vocabulario: ${ }^{2}$

1. Signos de conceptos: letras mayúsculas.

2. Signos distintivos de enunciados categóricos: $\{a, e, i, o\}$

3. Abreviatura de «es falso»: $\neg(.$.

- Expresiones bien formadas:

1.. Simples: un signo distintivo seguido inmediatamente por 2 signos de concepto. Por ejemplo: $« a \mathrm{BD} », \ll o \mathrm{BC} »$, etc. ${ }^{3}$ Se trata de una notación semejante a la propuesta por John Corcoran. ${ }^{4}$

2. si $\Gamma$ es una expresión simple bien formada, también lo es $\neg(\Gamma)$.

3. Por supuesto, no hay más expresiones correctas.

\section{I.2. EL FUNDAMENTO}

Leibniz propone el principio dictum de omni et nullo como fundamento de la lógica silogística, principio que expresa así:

Fundamentum Syllogisticum hoc est: Si totum aliquod C cadat intra aliquod $D$ vel si totum $C$ cadat extra aliquod D, tunc etiam id quod inest ipsi $C$ priore quidem casu cadet intra D, posteriore vero casu cadet extra D. Et hoc est quod vulgo vocant dictum de omni et nullo. ${ }^{5}$

1 Leibniz (1988): Opuscules et fragments inédits. Editado por Louis Couturat. Hildesheim, G. Olms, pp. 410-416.

2 A lo largo de este trabajo mantenemos los términos mnemotécnicos del propio Leibniz.

3 Leibniz (1988), p. 412.

$4 C f$. «Aristotle's Natural Deduction System» in Corcoran, J. (ed.) (1974): Ancient Logic and its Modern Interpretations. Dordrecht-Holanda, Reidel Publishing, pp. 85-131.

5 Leibniz (1988), pp. 410-411. 
Si se intenta dar expresión simbólica a este principio, se tiene:

$B$ inest $C$ si y sólo si, para cada $X$, si $X$ inest $B$ entonces $X$ inest $C$

Evidentemente, se cumple:

- $B$ inest $B^{6}$

- $\mathrm{Si}(B$ inest $C)$ y $(C$ inest $D)$ entonces $(B \text { inest } D)^{7}$

Entendemos que la expresión matemática correspondiente es:

$\Gamma \subseteq \Delta$ si y sólo si, para cada $X,{ }^{8}$ si $X \in \Gamma$ entonces $X \in \Delta$

Expresión que casi coincide plenamente con una definición exacta de inclusión como:

$\Gamma \subseteq \Delta$ si y sólo si, para cada $x \in A,{ }^{9}$ si $\mathrm{x} \in \Gamma$ entonces $\mathrm{x} \in \Delta$

Como es sabido, la relación de inclusión tiene 3 propiedades, a saber:

- reflexiva: $\Gamma \subseteq \Gamma$

- antisimétrica: $(\Gamma \subseteq \Delta) \wedge(\Delta \subseteq \Gamma) \rightarrow(\Gamma=\Delta)$ (que Leibniz no menciona en este trabajo ${ }^{10}$ )

- transitiva: $(\Gamma \subseteq \Delta) \wedge(\Delta \subseteq \Theta) \rightarrow(\Gamma \subseteq \Theta)$

\section{I.3. LOS CUATRO MODOS PERFECTOS}

Tomándolo por base, Leibniz establece el carácter básico de los cuatro modos perfectos de la primera figura: barbara, celarent, darii y ferio:

De aquí surgen inmediatamente los modos primitivos: todo $C$ es $D$. Todo $B$ es $C$. Luego todo B es D. $<$ o si se prefiere: todo B es C. Todo C es D. Luego todo B es D (esto es: los individuos de B mismo están contenidos entre los individuos de $\mathrm{C}$ y los individuos de $\mathrm{C}$ mismo están contenidos entre los individuos de D. Luego los individuos de B mismo están contenidos entre los individuos de D.> (esto es: todo el agregado de individuos de C están incluidos

6 Está propiedad da paso a las proposiciones que llama «idénticas» cuyo esquema es «a/i $\Gamma \Gamma » \mathrm{y}$ en los Nuevos Ensayos IV, 2 afirma que las proposiciones idénticas son primitivas de razón, necesarias por tanto.

7 Leibniz (1988), p. 411: «individua ipsius $B$ continentur in individuis ipsius $C$, et individua ipsius $C$ continentur in individuis ipsius $D$. Ergo individua ipsius $B$ continentur in individuis ipsius $D$ ». Véase también «Mathesis rationis», in Leibniz (1988), p. 194.

8 Entendemos que la $X$ puede ser sustituida por conceptos y sólo en el caso de especies últimas ha de ser sustituida por «cosas».

9 Donde $\mathbf{A}$ es el universo de discurso de una estructura dada $A$.

10 Corresponde al principio de los indiscernibles. Véase una formulación del mismo, por ejemplo, en la carta a Arnauld de 14 de julio de 1686. 
entre los individuos de D, y ya que todos los individuos de B están incluidos entre los individuos de $\mathrm{C}$, luego también entre los individuos de D).

Todo $C$ es $D$, algún $B$ es $C$, luego algún $B$ es $D .<0$ algún $B$ es C. Todo $C$ es D. Ergo algún B es D. (esto es: algunos individuos de B están contenidos entre los individuos de C. Todos los individuos de C están contenidos entre los individuos de D. Luego algunos individuos de B están contenidos entre los individuos de D).>

Ningún $C$ es $D$. Todo $B$ es $C$. Luego ningún $B$ es $D$. Y también ningún $C$ es D. Algún B es $C$. Luego algún B no es D. (Esto es: B o en su totalidad o en parte es-en $C$; ahora bien, $C$ entero cae fuera de D; luego $B$ o en su totalidad o en parte cae fuera de D. ${ }^{11}$

\section{I.4. TRANSFORMACIÓN DE LAS CONTRADICTORIAS}

Leibniz establece que si dos proposiciones son contradictorias (digamos: $\Gamma$ y $\neg \Gamma$, donde $\Gamma$ está por un enunciado categórico cualquiera), y si una es verdadera (si $\Gamma=1$ ), entonces la contradictoria es necesariamente falsa (entonces $\neg \Gamma=0)$ :

Hay contradicción entre la universal afirmativa y la particular negativa, o bien si es falsa $A, O$ es verdadera [regla $a \leftrightarrow o$ ]; y al contrario. Y también la hay entre la universal negativa y la particular afirmativa, o bien si es falsa $E$, $I$ es verdadera [regla $e \leftrightarrow i] .^{12}$

Lo que puede simbolizarse mediante las 2 reglas de transformación siguientes:

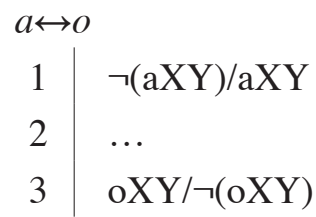

$$
\begin{array}{c|l}
e \leftrightarrow i & \\
1 & \neg(\mathrm{eXY}) / \mathrm{eXY} \\
2 & \ldots \\
3 & \mathrm{iXY} / \neg(\mathrm{iXY})
\end{array}
$$

\section{I.5. CONVERSIONES SIMPLES}

La demostración de los modos de la cuarta exige la incorporación de las conversiones: $e \leftrightarrow e$ y $i \leftrightarrow i$, cuyas demostraciones, según Leibniz, se obtienen así:

Para probar la cuarta figura se han de probar primero las conversiones simples:

11 Leibniz (1988), p. 411.

12 Leibniz (1988), pp. 412-413. 
1. En cesare (de la segunda) se demuestra que las universales negativas pueden ser convertidas simplemente: Ningún A es B; Todo B es B. Luego Ningún B es A.

2. En datisi (de la tercera) se demuestra que las particulares afirmativas pueden ser convertidas simplemente: Todo A es A; Algún A es B. Luego Algún B es A. ${ }^{13}$

Es decir:

\begin{tabular}{l|ll}
\multicolumn{3}{c}{$e \leftrightarrow e$} \\
1 & $\mathrm{eAB}$ & \\
2 & $\mathrm{aBB}$ & proposición idéntica \\
3 & $\mathrm{eBA}$ & cesare 1 y 2 \\
& \\
1 & aAA & proposición idéntica \\
2 & $\mathrm{iAB}$ & \\
3 & $\mathrm{iBA}$ & datisi 2 y 1
\end{tabular}

\section{I.6. SUBALTERNACIÓN}

A continuación, establece la validez de las relaciones de subalternación. Lo que resulta en las dos reglas siguientes: $a \rightarrow i$ y $e \rightarrow o$.

Subalternación (mediante la cual se deducen los otros dos modos de la primera figura a partir de los cuatro primitivos) se demuestra así: todo A es B. Algún A es A. Luego algún A es B. Que es un argumento en darii. Semejantemente: Ningún A es B. Algún A es A. Luego algún A no es B. Que es un argumento en ferio. ${ }^{14}$

Las pruebas son, según Leibniz, las siguientes:

\begin{tabular}{r|ll}
$a \rightarrow i$ & \\
1 & aAB & premisa \\
2 & iAA & proposición idéntica \\
3 & iAB & darii 1 y 2
\end{tabular}

13 Leibniz (1988), p. 416.

14 Leibniz (1988), p. 412. Aristóteles los justifica sin recurrir a ningún modo perfecto; los fundamenta mediante una prueba por reducción al absurdo. 


\begin{tabular}{l|ll}
$e \rightarrow o$ & \\
1 & eAB & premisa \\
2 & iAA & proposición idéntica \\
3 & oAB & ferio 1 y 2
\end{tabular}

\section{I.7. EL MÉTODO DEL REGRESSUS}

En el Regressus se usa este principio: si una conclusión es falsa (esto es: su contradictoria es verdadera) y una premisa es verdadera, entonces la otra premisa debe ser necesariamente falsa, o bien su contradictoria debe ser verdadera. Supone, pues, el Regressus el principio de contradicción. Hay contradicción entre la universal afirmativa y la particular negativa, o bien si es falsa $A, O$ es verdadera [regla a $\leftrightarrow$ o]; y al contrario. Y también la hay entre la universal negativa y la particular afirmativa, o bien si es falsa $E, I$ es verdadera [regla e↔i]. ${ }^{15}$

Este principio puede remontarse a los estoicos, quienes lo expresan mediante una meta-regla semántica: «Si de dos proposiciones se deduce una tercera, entonces cualquiera de las dos junto con la negación de la conclusión produce la negación de la otra». ${ }^{16}$

Couturat lo entiende como una técnica de prueba por reducción al absurdo: «Para reducir los modos de las tres últimas figuras a los de la primera, conforme a la tradición de Aristóteles, Leibniz adopta un método ingenioso que le había sido sugerido por su maestro Jacob Thomasius pero cuya invención se remonta a Ramus. Leibniz llama a este método regressus y nosotros reducción al absurdo». ${ }^{17}$ Con lo que nos podríamos remontar al propio Aristóteles quien ya sostuvo que es posible demostrar la validez de un modo legítimo de la 2. o 3. ${ }^{\text {a }}$ por reducción al absurdo. ${ }^{18}$

Leibniz proporciona en un cuadro la aplicación de este método a los modos de la segunda y tercera figura. Copiamos una parte de ese cuadro: ${ }^{19}$

15 Leibniz (1988), pp. 412-413.

16 Mates, B. (1985): La lógica de los estoicos. Traducción de M. García Baró. Madrid, Tecnos. Pág. 132 y ss.

17 Louis Couturat (1969): La logique de Leibniz. Hildesheim, Georg Olms. Pág. 8.

18 Cf. Analíticos primeros I, 29, 45a 24 y ss.

19 Leibniz (1988), pp. 413-414. 
Cuadro 1: Aplicación del Regressus por Leibniz

\begin{tabular}{|c|c|c|c|}
\hline \multicolumn{3}{|c|}{ expresiones } & expresiones \\
\hline barbara primae & ACD . ABC . ABD & barbara primae & ACD . ABC . ABD \\
\hline Regressus & ACD . OBC . OBD & Regressus & OCD . ABC . OBD \\
\hline baroco secundae & ACD . OBD . OBC & bocardo tertiae & OBD . ABC . OCD \\
\hline celarent primae & ECD . ABC . EBD & celarent primae & ECD . ABC . EBD \\
\hline Regressus & ECD . OBC . IBD & Regressus & ICD . ABC . IBD \\
\hline festino secundae & ECD . IBD . OBC & disamis tertiae & IBD . ABC . ICD \\
\hline
\end{tabular}

\section{SOBRE LOS FUNDAMENTOS}

\section{II.1. EL DICTUM DE OMNI Y LOS MODOS PERFECTOS}

Como es sabido, Aristóteles ensaya una justificación de los modos perfectos en el capítulo 4 del libro primero de los Analíticos primeros. Por «justificación» hay que entender, según creemos, intentar probar que cada uno de ellos es «verdadero». Hoy diríamos, quizás, «correcto» porque su aplicación preserva la verdad de los enunciados a los que se aplique para obtener una conclusión inmediata. Pero se trata de una prueba. Lo que hace Aristóteles puede analizarse en el caso de barbara. Aristóteles lo enuncia como sigue:

Si A se predica de todo ${ }^{20} \mathrm{~B}$ y B se predica de todo $\mathrm{C}$, necesariamente A se predica de todo C. (I, 4, 25b39-41)

Reparemos, ante todo, en que Aristóteles le da forma de un único enunciado. $\mathrm{Su}$ aspecto deja ver una cierta transitividad: $\mathrm{A} \rightarrow \mathrm{B}, \mathrm{B} \rightarrow \mathrm{C}, \mathrm{A} \rightarrow \mathrm{C}$.

En los Analíticos segundos, que contiene su teoría del método deductivo, Aristóteles afirma que los principios han de ser indemostrables. Pero no se queda ahí y pide para todo lo que tenga carácter de algo «anterior»-términos primitivos, axiomas- un fundamento que a su juicio se logra en virtud de otra función mental distinta de la ilación o razonamiento. ¿Por qué esta exigencia de fundamento? Porque los axiomas han de ser más verdaderos que cualquier otra verdad probada. Y dicha verdad, puesto que no puede estar fundada en ninguna anterior, ha de ser inmediata:

Si el conocimiento es como se ha dicho, es necesario para cualquier conocimiento demostrativo basarse en cosas más verdaderas, primeras, inmediatas y conocidas que, y que sean causa de, la conclusión. $(2,71 \mathrm{~b} 20-$ 23)

20 Katà pantós. 
No obstante, Aristóteles da una justificación. En efecto, en el modo barbara hay, al igual que en los demás, 3 términos predicativos. Aristóteles examina las relaciones entre estos tres términos. Sean $A, B$ y $C$. Si $C$ está incluido en $B$ y $B$ está incluido en $A$, necesariamente $C$ está incluido en $A$. Y lo dice así:

Así, pues, cuando tres términos se relacionan entre sí de tal manera que el último esté $<$ incluido $>$ en el conjunto del $<$ término $>$ medio y el $<$ término $>$ medio esté o no esté $<$ incluido $>$ en el conjunto del $<$ término $>$ primero, habrá necesariamente un silogismo perfecto entre los <términos $>$ extremos. ( $A n$. $\operatorname{Pr}$. I, 4, 25b32-35. Sustituimos «contenido» por «incluido» $\mathrm{y}$ «razonamiento» por «silogismo» en la trad. de Candel.)

Ahora bien, como predicarse «katà pantós» significa lo mismo que estar incluido en:

Que una cosa esté incluida en otra es lo mismo que decir que una cosa es predicada «katà pantós» de otra. Usamos «katà pantós» cuando no se puede encontrar en el sujeto ninguna parte de la que no se puede afirmar el predicado; usamos «katà medenós» de modo semejante. ${ }^{21}$

25b32-35 ha de entenderse como una justificación de lo dicho en la primera cita.

Leibniz advierte que el fundamento último de la corrección de barbara está en las relaciones de «inclusión» entre los 3 términos existentes en barbara. E identifica dicho fundamento con el principio dictum de omni et nullo. A su juicio, este principio establece cuándo hay relación entre dos conceptos: «Si totum aliquod $C$ cadat intra aliquod $D$...id quod inest ipsi $C$ cadet intra $D »$. Idea que hemos entendido del modo siguiente:

$B$ inest $C$ si y sólo si, para cada $X$, si $X$ inest $B$ entonces $X$ inest $C$

donde $X$ no ha de ser entendida como «cosa» sino como otro concepto. Así, por ejemplo, si triángulo rectángulo tiene 2 especies: de catetos iguales y de catetos desiguales, la extensión de triángulo rectángulo está constituida exclusivamente por estos dos conceptos. Y por ello, cualquier propiedad que se diga de triángulo rectángulo se dice de cada uno de los elementos de su extensión. Sin embargo, cuando un concepto es una especie última, su extensión no puede estar formada por conceptos sino por «cosas». Evidentemente de cada una de estas cosas se dice con verdad la especie última y cada propiedad que está tenga conviene también a cada una de esas «cosas».

Pero Leibniz, como antes Aristóteles, no advierte que hay que dar un sentido único o último a la idea de extensión. Y dado que en el caso de las especies 
últimas «extensión» sólo puede significar un conjunto de «cosas», y que no puede significar un conjunto de otros conceptos, y que en el caso de otra especie no última la extensión puede determinarse también mediante un conjunto de «cosas», la idea de «extensión de un concepto» sólo puede unificarse mediante la idea de un conjunto de «cosas», es decir extensionalmente. Pero lo cierto es que tanto Aristóteles como Leibniz dejan este problema sin resolver.

Con independencia de la precisión de la idea de extensión, parece claro que tanto Leibniz como Aristóteles establecen como fundamento último de los modos perfectos la relación de «inclusión» entre conceptos. Leibniz aísla este fundamento y lo formula como principio de la corrección o verdad de los modos perfectos. El dictum de omni et nullo, pues, fija y define el concepto de inclusión, si bien sin terminar de precisar el de extensión de un concepto. O, si se prefiere, sin advertir que la idea de inclusión presupone la de pertenencia de un elemento a un conjunto y ésta exige la idea de universo de discurso.

Con esto, Leibniz puede convertir cada uno de los cuatro modos básicos en una «regla básica» de un cálculo.

\section{II.2. DE LAS CONTRADICTORIAS, CONVERSIÓN SIMPLE Y SUBALTERNACIÓN}

Aristóteles usa, en la prueba por conversión de un modo de la segunda o tercera, reglas: de la transformación de contradictorias, de la transformación simple de universales negativos (E) y de particulares afirmativos (I), y de la subalternación -en particular la transformación de las universales afirmativas (A) en particulares afirmativas (I). Pero Aristóteles tiene sumo cuidado en justificar estas reglas aparte de cualquier silogismo.

El proceder de Leibniz es distinto. La transformación de las contradictorias es fundada en el principio de bivalencia. Él dice en el principio de contradicción. La idea es muy sencilla: si sólo hay dos valores de verdad y un enunciado « $X$ » es verdadero, su contradictoria $« \neg X »$ ha de ser falsa. De aquí sale la verdad o corrección de las reglas de transformación de las contradictorias.

Sin embargo, tanto las reglas de la conversión simple cuanto las de la subalternación son justificadas mediante un silogismo en el que se hace uso de algún modo perfecto o, incluso, de algún modo imperfecto considerado como regla derivada. En este punto hay algo de precipitación en Leibniz. En efecto, si se fundan los modos perfectos en el principio dictum de omni y la transformación de las contradictorias en el de bivalencia, se pueden tomar estas reglas como «básicas». Y, partiendo de ellas, es posible obtener la fundamentación de las reglas de conversión simple, como hace el propio Leibniz en la fundamentación de la subalternación, del modo siguiente: 


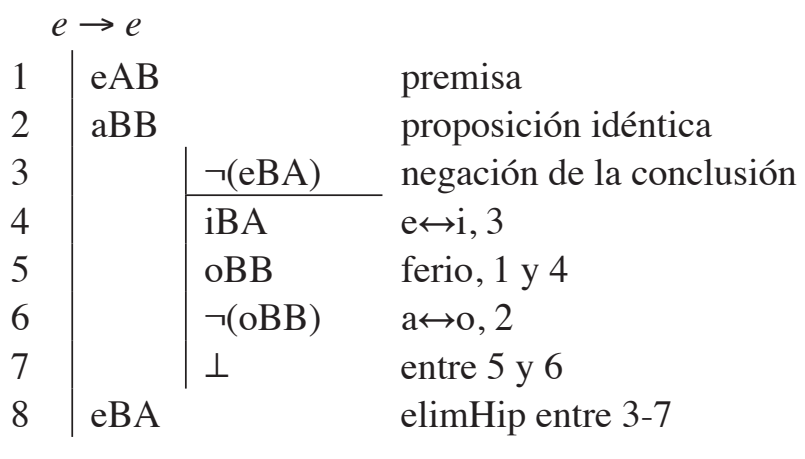

\begin{tabular}{l|l|ll}
\multicolumn{2}{c}{$i \rightarrow i$} & & \\
1 & $\mathrm{iAB}$ & & premisa \\
2 & $\mathrm{aBB}$ & & proposición idéntica \\
3 & & $\neg(\mathrm{iBA})$ & negación de la conclusión \\
4 & & $\mathrm{eBA}$ & $\mathrm{e} \leftrightarrow \mathrm{i}, 3$ \\
5 & & oAA & ferio, 4 y 1 \\
6 & & $\neg(\mathrm{oAA})$ & a $\leftrightarrow \mathrm{O}, 2$ \\
7 & & $\perp$ & entre 5 y 6 \\
8 & iBA & & elimHip entre 3-7
\end{tabular}

\section{II.3. ORdENACIÓN DE LAS REGLAS DEL CÁlCULO}

De modo que hay que presentar las reglas del cálculo propuesto del modo siguiente:

1. Reglas básicas:

- Reglas de los modos perfectos:

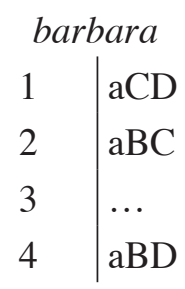

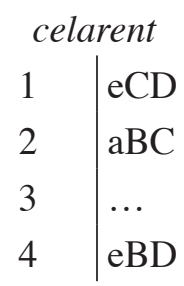

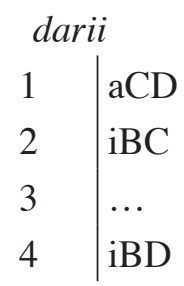

\begin{tabular}{l|l} 
ferio \\
1 & $\mathrm{eCD}$ \\
2 & $\mathrm{iBC}$ \\
3 & $\ldots$ \\
4 & $\mathrm{oBD}$
\end{tabular}


- Reglas de las contradictorias:

\begin{tabular}{l|ll|l}
$a \leftrightarrow o$ & \multicolumn{3}{|c}{$e \leftrightarrow i$} \\
1 & $\neg(\mathrm{aXY}) / \mathrm{aXY}$ & 1 & $\neg(\mathrm{eXY}) / \mathrm{eXY}$ \\
2 & $\ldots$ & 2 & $\ldots$ \\
3 & $\mathrm{oXY} / \neg(\mathrm{oXY})$ & 3 & $\mathrm{iXY} / \neg(\mathrm{iXY})$
\end{tabular}

- Eliminación de la hipótesis: sea $\Gamma$ una expresión simple:

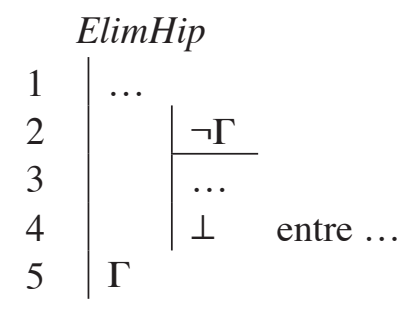

2. Reglas derivadas: sean $\Gamma$ y $\Delta$ símbolos de conceptos

- Reglas de la subalternación:

\begin{tabular}{|c|c|c|c|}
\hline \multicolumn{2}{|c|}{$a \rightarrow i$} & \multicolumn{2}{|c|}{$e \rightarrow o$} \\
\hline 1 & $\mathrm{a} \Gamma \Delta$ & 1 & $\mathrm{i} \Gamma \Delta$ \\
\hline 2 & $\ldots$ & 2 & $\cdots$ \\
\hline 3 & $\mathrm{e} \Gamma \Delta$ & 3 & $\mathrm{i} \Gamma \Delta$ \\
\hline
\end{tabular}

- Reglas de la conversión simple:

\begin{tabular}{l|ll|l}
$e \rightarrow e$ & \multicolumn{3}{c}{$i \rightarrow i$} \\
1 & $\mathrm{e} \Gamma \Delta$ & 1 & $\mathrm{i} \Gamma \Delta$ \\
2 & $\ldots$ & 2 & $\ldots$ \\
3 & $\mathrm{e} \Delta \Gamma$ & 3 & $\mathrm{i} \Delta \Gamma$
\end{tabular}

III. REGRESSUS Y REDUCCIÓN AL ABSURDO

Pero si tomamos la interpretación de Couturat al pie de la letra y el método del regressus es entendido como un procedimiento de prueba mediante una 
estrategia de reducción al absurdo, entonces hay que introducir algunas modificaciones en el cálculo anterior. ${ }^{22}$ En concreto hay que hacer así:

\section{III.1. NOTACIÓN, REGLAS DE FORMACIÓN Y REGLAS DEL CÁLCULO}

- Vocabulario:

1. Signos de conceptos: letras mayúsculas.

2. Signos distintivos de enunciados categóricos: $\{a, e, i, o\}$

3. Abreviatura de «es falso»: $\neg(.$.

4. $\perp$ abreviatura de hay una contradicción entre ...

- Expresiones bien fomadas:

1. Simples: un signo distintivo seguido inmediatamente por 2 signos de concepto. Por ejemplo: $« a \mathrm{BD} », \ll o \mathrm{BC} »$, etc..

2. Si $\Gamma$ es una expresión simple bien formada, también lo es $\neg(\Gamma)$.

3. Por supuesto, no hay más expresiones correctas.

- Reglas del cálculo:

- Reglas de los modos perfectos:
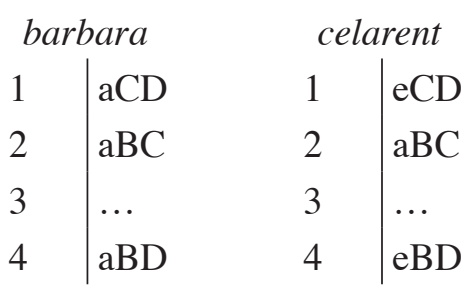

\begin{tabular}{l|l}
\multicolumn{2}{c}{ darii } \\
1 & $\mathrm{aCD}$ \\
2 & $\mathrm{iBC}$ \\
3 & $\ldots$ \\
4 & $\mathrm{iBD}$
\end{tabular}

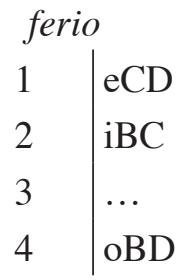

- Reglas de las contradictorias:

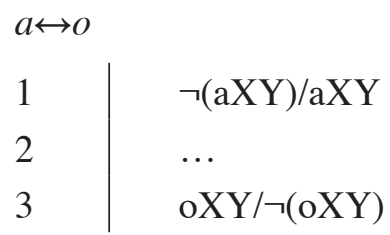

$$
\begin{array}{l|l}
e \leftrightarrow i & \\
1 & \neg(\mathrm{eXY}) / \mathrm{eXY} \\
2 & \ldots \\
3 & \mathrm{iXY} / \neg(\mathrm{iXY})
\end{array}
$$

22 Ya hemos dicho que la idea está en el propio Aristóteles, Analíticos primeros I, 29, $45 \mathrm{a} 24$ y ss. 
- Reglas de la conversión: sean $\Gamma$ y $\Delta$ símbolos de conceptos

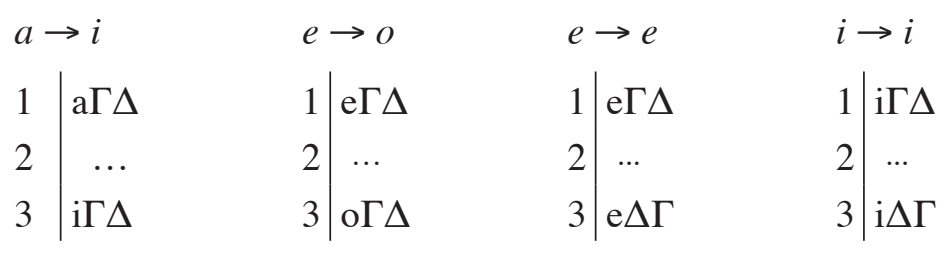

- Eliminación de la hipótesis: sea $\Gamma$ una expresión simple

\section{ElimHip}

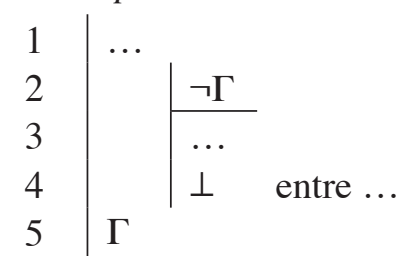

III.2. REGRESSUS EN CUANTO ESTRATEGIA DE REDUCCIÓN AL ABSURDO

En líneas generales se trata de plantear una deducción de la siguiente manera:

1. Se afirman las dos premisas;

2. Se supone la negación de la conclusión;

3. Se eliminará esta hipótesis alcanzando una contradicción. Lo que se logrará:

(a) Aplicando un modo perfecto;

(b) Transformando una de las dos premisas según alguna de las reglas de las contradictorias;

(c) En algunas deducciones habrá que aplicar, además, reglas de conversión;

4. Si se ha logrado lo anterior, se afirma la conclusión. 


\section{III.3. EJEMPLOS}

baroco (2. ${ }^{\text {a }}$ figura)

\begin{tabular}{|c|c|c|c|}
\hline 1 & $\mathrm{aPM}$ & & primera premisa \\
\hline 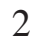 & oSM & & segunda premisa \\
\hline 3 & & $\neg(\mathrm{oSP})$ & negación de la conclusión \\
\hline 4 & & aSP & $a \leftrightarrow o, 3$ \\
\hline 5 & & aSM & barbara, 1 y 4 \\
\hline 6 & & $\neg(\mathrm{aSM})$ & $a \leftrightarrow o, 2$ \\
\hline 7 & & $\perp$ & entre 5 y 6 \\
\hline 8 & oSP & & elimHip entre $3-7$ \\
\hline
\end{tabular}

bocardo (3. ${ }^{\text {a }}$ figura)

\begin{tabular}{|c|c|c|c|}
\hline 1 & oMP & & primera premisa \\
\hline 2 & aMS & & segunda premisa \\
\hline 3 & & $\neg(\mathrm{oSP})$ & negación de la conclusión \\
\hline 4 & & aSP & $a \leftrightarrow o, 3$ \\
\hline 5 & & aMP & barbara, 4 y 2 \\
\hline 6 & & $\neg(\mathrm{aMP})$ & $a \leftrightarrow o, 1$ \\
\hline 7 & & $\perp$ & entre 5 y 6 \\
\hline & oSP & & elimHip entre 3-7 \\
\hline
\end{tabular}

\begin{tabular}{l|lll}
\multicolumn{3}{l}{ calerent (4. a $^{\text {figura })}$} & \\
1 & aPM & & primera premisa \\
2 & eMS & & segunda premisa \\
3 & & $\neg(\mathrm{eSP})$ & negación de la conclusión \\
4 & & iSP & $e \leftrightarrow i, 3$ \\
5 & & iSM & darii, 1 y 4 \\
6 & & iMS & $i \rightarrow i, 5$ \\
7 & & $\neg(\mathrm{iMS})$ & $e \leftrightarrow i, 2$ \\
8 & & $\perp$ & entre 6 y 7 \\
9 & eSP & & elimHip entre 3-8
\end{tabular}




\section{REGRESSUS COMO UN ALGORITMO DE PRODUCCIÓN}

Leibniz, a nuestro juicio, entiende el cálculo del Regressus como una técnica algorítmica que produce, partiendo de un modo de la primera, un modo igualmente legítimo de la segunda o tercera figura, cuyo fundamento semántico es el siguiente: si $(\Gamma \wedge \Delta) \vDash \Theta$, se cumplirá que en aquellas interpretaciones en que $\Gamma$ es verdadera y $\Theta$ es falsa, $\Delta$ ha de ser necesariamente falsa. Y también que en aquellas en que $\Delta$ es verdadera y $\Theta$ es falsa, $\Gamma$ ha de ser necesariamente falsa. Por tanto, serán verdad:

$$
\begin{aligned}
& \text { si } \Gamma \wedge \Delta \vDash \Theta \text { entonces } \Gamma \wedge \neg \Theta \vDash \neg \Delta \\
& \text { si } \Gamma \wedge \Delta \vDash \Theta \text { entonces } \neg \Theta \wedge \Delta \vDash \neg \Gamma .
\end{aligned}
$$

El procedimiento consta de dos pasos. Dado un modo legítimo de la primera figura:

- PARA PRODUCIR UN MODO LEGÍTIMO DE LA SEGUNDA:

1. se mantiene la primera premisa.

2. se afirma la contradictoria de la conclusión mediante la aplicación de una de las dos reglas de transformación de las contradictorias.

3. se concluye afirmando la contradictoria de la segunda premisa mediante aplicación de una de las dos reglas de transformación de las contradictorias.

Por ejemplo:

de barbara

$$
\begin{array}{l|ll}
1 & \mathrm{aCD} & 1 .{ }^{\mathrm{a}} \text { premisa barbara } \\
2 & \mathrm{aBC} & 2 .{ }^{\mathrm{a}} \text { premisa barbara } \\
3 & \mathrm{aBD} & \text { conclusión barbara }
\end{array}
$$

se genera baroco, de la $2 .^{\mathrm{a}}$

\begin{tabular}{l|ll}
1 & $\mathrm{aCD}$ & $1 .^{\mathrm{a}}$ premisa barbara \\
2 & $\neg(\mathrm{aBD})$ & conclusión es falsa \\
3 & $\mathrm{oBD}$ & $\mathrm{a} \leftrightarrow \mathrm{o}$ \\
4 & $\neg(\mathrm{aBC})$ & $2 .^{\mathrm{a}}$ premisa es falsa \\
5 & $\mathrm{oBC}$ & $\mathrm{a} \leftrightarrow \mathrm{O}$
\end{tabular}


- PARA PROdUCIR UN MOdo LeGítimo de la terCera:

1. se afirma la contradictoria de la conclusión mediante la aplicación de una de las dos reglas de transformación de las contradictorias 2. se mantiene la segunda premisa

3. se concluye afirmando la contradictoria de la primera premisa mediante aplicación de una de las dos reglas de transformación de las contradictorias

de barbara

Por ejemplo:

1 aCD $1 .^{\text {a }}$ premisa barbara

2 aBC 2. ${ }^{\text {a }}$ premisa barbara

3 aBD conclusión barbara

se genera bocardo de la tercera

\begin{tabular}{l|ll}
1 & $\neg(\mathrm{aBD})$ & conclusión es falsa \\
2 & $\mathrm{oBD}$ & $a \leftrightarrow o$ \\
3 & $\mathrm{aBC}$ & $2 .^{\mathrm{a}}$ premisa \\
4 & $\neg(\mathrm{aCD})$ & $1 .^{\mathrm{a}}$ premisa es falsa \\
5 & $\mathrm{oCD}$ & $a \leftrightarrow o$
\end{tabular}

\section{IV.1. Regressus COMO UN SISTEMA À LA POST}

Como es sabido, Emil Post (1943 23 ) sostuvo que cualquier sistema de cálculo es reducible en último extremo a una manipulación reglada de ristras de signos de un alfabeto finito. Esta idea se tradujo en los conocidos sistemas canónicos o sistemas de reglas de producción. ${ }^{24}$ Creemos que es posible expresar la idea anterior en el siguiente sistema canónico:

1. Alfabeto $=\{a, e, i, o, \neg, \mathrm{M}, \mathrm{P}, \mathrm{S},$.

2. Axiomas:

(a) aMP.aSM .aSP

(b) eMP.aSM.eSP

$23 C f$. «Formal Reductions of the General Combinatorial Decision Problem», en Martin Davis (ed) (1994): Solvability, Provability, Definability: The Collected Works of Emil L. Post. Boston, Birkhäuser, pp. 442-460.

24 Cf. Marvin Minsky (1967): Computation: Finite and Infinite Machines. Englewood Cliffs (N. J.), Prentice-Hall. Cap. 12. 
(c) aMP.iSM.iSP

(d) $e M P . i S M . o S P$

3. Reglas de producción (donde $X$ e $Y$ están por uno de los signos M, P o S):
(a) R1: $\neg a X Y \rightarrow o X Y$
(b) R2: $\neg o X Y \rightarrow a X Y$
(c) R3: $\neg e X Y \rightarrow i X Y$
(d) R4: $\neg i X Y \rightarrow e X Y$
(e) D1: $a X Y \rightarrow i X Y$
(f) D2: $e X Y \rightarrow o X Y$

4. Esquemas de Teoremas (donde $P_{1}$ está por la primera premisa, $P_{2}$ por la segunda y $C$ por la conclusión de un axioma o teorema):
(a) $P_{1} \cdot P_{2} \cdot C \rightarrow P_{1} \cdot P_{2} \cdot D_{\mathrm{i}}(C)$
(b) $P_{1} \cdot P_{2} \cdot C \rightarrow P_{1} \cdot R_{\mathrm{i}}(\neg C) \cdot R_{\mathrm{i}}\left(\neg P_{2}\right)$
(c) $P_{1} \cdot P_{2} \cdot C \rightarrow R_{\mathrm{i}}(\neg C) \cdot P_{2} \cdot R_{\mathrm{i}}\left(\neg P_{1}\right)$

\section{IV.2. PRODUCCIÓN DE LOS MODOS IMPERFECTOS LEGÍTIMOS}

Aplicando uno de los esquemas de teorema, un axioma o un teorema de la primera figura, junto con una o más reglas de producción, es posible obtener, por mera transformación sígnica, cada uno de los modos legítimos de la segunda y tercera figura. Los cuadros siguientes lo muestran.

Cuadro 2: Teoremas de la 1. a figura

\begin{tabular}{|c|c|c|}
\hline Esquema & Axioma & Producción \\
\hline$P_{1} . P_{2} . C \rightarrow P_{1} . P_{2} . D c(C)$ & aMP.aSM.aSP & $\begin{array}{r}\text { aMP.aSM.aSP } \rightarrow a M P . a S M . D 1(a S P) \\
=a M P . a S M . i S P\end{array}$ \\
\hline$P_{1} . P_{2} . C \rightarrow P_{1} . P_{2} . D c(C)$ & eMP.aSM.eSP & $\begin{array}{r}\text { eMP.aSM.eSP } \rightarrow e M P . a S M . D 2(e S P) \\
=e M P . a S M . o S P\end{array}$ \\
\hline
\end{tabular}


Cuadro 3: Teoremas de la 2. ${ }^{\text {a figura }}$

\begin{tabular}{|c|c|c|}
\hline Esquema & Axioma & Producción \\
\hline$P_{1} \cdot P_{2} \cdot \mathrm{C} \rightarrow P_{1} \cdot R_{\mathrm{i}}(\neg C) \cdot R_{\mathrm{i}}\left(\neg P_{2}\right)$ & aMP.aSM.aSP & $\begin{aligned} \text { aMP.aSM.aSP } & \rightarrow a M P . R 1(\neg a S P) \cdot R 1(\neg a S M) \\
& =a M P . o S P . o S M\end{aligned}$ \\
\hline$P_{1} \cdot P_{2} \cdot C \rightarrow P_{1} \cdot R_{i}(\neg C) \cdot R_{i}\left(\neg P_{2}\right)$ & eMP.aSM.eSP & $\begin{aligned} e M P . a S M . e S P & \rightarrow e M P . R 3(\neg e S P) . R 1(\neg a S M) \\
& =e M P . i S P . o S M\end{aligned}$ \\
\hline$P_{1} \cdot P_{2} \cdot C \rightarrow P_{1} \cdot R_{i}(\neg C) \cdot R_{i}\left(\neg P_{2}\right)$ & aMP.iSM.iSP & $\begin{aligned} a M P . i S M . i S P & \longrightarrow a M P . R 4(\neg i S P) . R 4(\neg i S M) \\
& =a M P . e S P . e S M\end{aligned}$ \\
\hline$P_{1} \cdot P_{2} \cdot C \rightarrow P_{1} \cdot R_{i}(\neg C) \cdot R_{i}\left(\neg P_{2}\right)$ & eMP.iSM.oSP & $\begin{aligned} e M P . i S M . o S P & \rightarrow e M P . R 2(\neg o S P) . R 4(\neg i S M) \\
& =e M P . a S P . e S M\end{aligned}$ \\
\hline Esquema & Teorema $1 .^{\mathrm{a}}$ & Producción \\
\hline$P_{1} \cdot P_{2} \cdot C \rightarrow P_{1} \cdot R_{i}(\neg C) \cdot R_{i}\left(\neg P_{2}\right)$ & $a M P . a S M . i S P$ & $\begin{aligned} a M P . a S M . i S P & \rightarrow a M P . R 4(\neg i S P) . R 1(\neg a S M) \\
& =a M P . e S P . o S M\end{aligned}$ \\
\hline$P_{1} \cdot P_{2} \cdot C \rightarrow P_{1} \cdot R_{i}(\neg C) \cdot R_{i}\left(\neg P_{2}\right)$ & eMP.aSM.oSP & $\begin{aligned} e M P . a S M . o S P & \rightarrow e M P . R 2(\neg o S P) . R 1(\neg a S M) \\
& =e M P . a S P . o S M\end{aligned}$ \\
\hline
\end{tabular}

Cuadro 4: Teoremas de la 3. ${ }^{\text {figura }}$

\begin{tabular}{|c|c|c|}
\hline Esquema & Axioma & Producción \\
\hline$P_{1} \cdot P_{2} \cdot C \rightarrow R_{i}(\neg C) \cdot P_{2} \cdot R_{i}\left(\neg P_{1}\right)$ & $a M P . a S M . a S P$ & $\begin{aligned} a M P . a S M \cdot a S P & \longrightarrow R 1(\neg a S P) \cdot a S M \cdot R 1(\neg a M P) \\
= & o S P \cdot a S M \cdot o M P\end{aligned}$ \\
\hline$P_{1} \cdot P_{2} \cdot C \rightarrow R_{i}(\neg C) \cdot P_{2} \cdot R_{i}\left(\neg P_{1}\right)$ & eMP.aSM.eSP & $\begin{aligned} e M P . a S M . e S P & \rightarrow R 3(\neg e S P) \cdot a S M \cdot R 3(\neg e M P) \\
& =i S P \cdot a S M \cdot i M P\end{aligned}$ \\
\hline$P_{1} \cdot P_{2} \cdot C \rightarrow R_{i}(\neg C) \cdot P_{2} \cdot R_{i}\left(\neg P_{1}\right)$ & $a M P . i S M . i S P$ & $\begin{aligned} a M P . i S M . i S P & \rightarrow R 4(\neg i S P) . i S M . R 1(\neg a M P) \\
& =e S P . i S M . o M P\end{aligned}$ \\
\hline$P_{1} \cdot P_{2} . C \rightarrow R_{i}(\neg C) \cdot P_{2} \cdot R_{i}\left(\neg P_{l}\right)$ & eMP.iSM.oSP & $\begin{aligned} e M P . i S M . o S P & \rightarrow R 2(\neg o S P) . i S M . R 3(\neg e M P) \\
& =a S P . i S M . i M P\end{aligned}$ \\
\hline Esquema & Teorema $1 .^{a}$ & Producción \\
\hline$P_{1} \cdot P_{2} \cdot C \rightarrow R_{i}(\neg C) \cdot P_{2} \cdot R_{i}\left(\neg P_{1}\right)$ & aMP.aSM.iSP & $\begin{aligned} a M P . a S M . i S P & \longrightarrow R 4(\neg i S P) . a S M \cdot R 1(\neg a M P) \\
& =e S P . a S M . o M P\end{aligned}$ \\
\hline$P_{1} \cdot P_{2} \cdot C \rightarrow R_{i}(\neg C) \cdot P_{2} \cdot R_{i}\left(\neg P_{1}\right)$ & eMP.aSM.oSP & $\begin{aligned} e M P . a S M . o S P & \rightarrow R 2(\neg o S P) . a S M \cdot R 3(\neg e M P) \\
& =a S P . a S M . i M P\end{aligned}$ \\
\hline
\end{tabular}

\section{Un MÉTODO DE DECISIÓN FUNDADO EN EL REGRESSUS}

Como en cualquier otra lógica, un problema en la lógica silogística se plantea en términos de si una inferencia es legítima. Es decir, el problema es averiguar si el esquema de un modo es válido o no. Continuando la idea de Leibniz, es posible «invertir el Regressus». Esto es, partir del modo problema dado, identificar a qué figura pertenece e intentar reducirlo a una ley lógica. La idea central es ésta: 


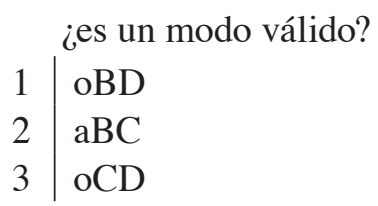

Figura del esquema anterior: 3 . $^{\text {a }}$

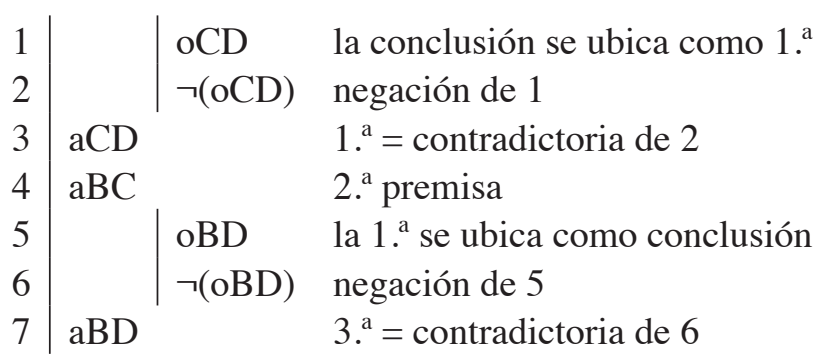

Si el esquema resultante es una de las 4 leyes lógicas, el modo es válido.

La explicación de esta idea es la siguiente:

- Si el modo problema fuera legítimo, se podría obtener por producción a partir de una ley de la 1. a figura.

- El procedimiento de producción depende de la figura a la que corresponda el modo problema.

- En el ejemplo anterior, la figura es la 3..

- Luego habría que hacer lo siguiente:

Dado que:

1. la primera premisa se produce a partir de la negación de la conclusión de la ley de la 1. figura,

2. la segunda premisa coincide con la segunda premisa de la ley de la 1. ${ }^{\text {a figura } \mathrm{y}}$

3. la conclusión se produce a partir de la negación de la primera premisa de la ley de la 1. figura,

Entonces:

1. la primera premisa de la ley lógica se ha de poder obtener aplicando una regla de la contradicción a la negación de la conclusión del modo problema,

2. la segunda premisa de la ley se ha de poder obtener escribiendo sin más la segunda premisa del modo problema y

3. la conclusión de la ley se ha de poder obtener aplicando una regla de la contradicción a la negación de la primera premisa del modo problema. 
Intentaremos presentar esta idea mediante un sistema canónico en la siguiente sección.

\section{V.1. DECISIÓN À LA POST}

El punto fundamental es sustituir en el anterior sistema canónico los esquemas de teoremas. La idea es que en la parte antecedente aparezca el modo problema, mientras que en la parte consecuente se transforma de modo tal que se produzca o un axioma o un teorema de la 1. figura.

1. Alfabeto $=\{a, e, i, o, \neg, \mathrm{M}, \mathrm{P}, \mathrm{S},$.

2. Esquemas de Axiomas (donde $X, Y$ y $Z$ están por uno de los signos M, P o S):
(a) aZY.aXZ.aXY
(b) eZY.aXZ.eXY
(c) $a Z Y . i X Z . i X Y$
(d) eZY.iXZ.oXY

3. Reglas de producción (donde $X$ e $Y$ están por uno de los signos M, P o S):
(a) R1: $\neg a X Y \rightarrow o X Y$
(b) R2: $\neg o X Y \rightarrow a X Y$
(c) R3: $\neg e X Y \rightarrow i X Y$
(d) R4: $\neg i X Y \rightarrow e X Y$
(e) ID1: $i X Y \rightarrow a X Y$
(f) ID2: $o X Y \rightarrow e X Y$

4. Esquemas de Decisión (donde $P_{1}$ está por la primera premisa, $P_{2}$ por la segunda y $C$ por la conclusión del modo problema):

(a) $P_{1} \cdot P_{2} \cdot C \rightarrow P_{1} \cdot R_{i}(\neg C) \cdot R_{i}\left(\neg P_{2}\right)$

(b) $P_{1} \cdot P_{2} \cdot C \rightarrow R_{i}(\neg C) \cdot P_{2} \cdot R_{i}\left(\neg P_{1}\right)$

Cuadro 5: Ejemplos de problemas

\begin{tabular}{|c|c|c|}
\hline Modo problema & Esquema de decisión & Producción \\
\hline oMP.aMS.oSP & $P_{1} \cdot P_{2} \cdot C \rightarrow R_{i}(\neg C) \cdot P_{2} \cdot R_{i}\left(\neg P_{1}\right)$ & $\begin{array}{c}o M P . a M S . o S P \rightarrow R 2(\neg O S P) \cdot a M S . R 2(\neg o M P) \\
=a S P . a M S \cdot a M P(\text { barbara })\end{array}$ \\
\hline aPM.eSM.eSP & $P_{1} \cdot P_{2} \cdot C \rightarrow P_{1} \cdot R_{i}(\neg C) \cdot R_{i}\left(\neg P_{2}\right)$ & $\begin{array}{c}\text { aPM.eSM.eSP } \rightarrow a P M . R 3(\neg e S P) \cdot R 3(\neg e S M) \\
=a P M . i S P . i S M(\text { darii) }\end{array}$ \\
\hline eMP.iMS.oSP & $P_{1} \cdot P_{2} \cdot C \rightarrow R_{i}(\neg C) \cdot P_{2} \cdot R_{i}\left(\neg P_{1}\right)$ & $\begin{array}{c}e M P . i M S . o S P \rightarrow R 2(\neg o S P) . i M S . R 3(\neg e M P) \\
=a S P . i M S . i M P(\text { darii })\end{array}$ \\
\hline ePM.aSM.eSM & $P_{1} \cdot P_{2} \cdot C \rightarrow P_{1} \cdot R_{i}(\neg C) \cdot R_{i}\left(\neg P_{2}\right)$ & $\begin{array}{c}\text { ePM.aSM.eSM } \rightarrow e P M . R 3(\neg e S M) \cdot R 1(\neg a S M) \\
=e P M . i S M . o S M(\text { ferio })\end{array}$ \\
\hline oPM.oSM.aSP & $P_{1} \cdot P_{2} \cdot C \rightarrow P_{1} \cdot R_{i}(\neg C) \cdot R_{i}\left(\neg P_{2}\right)$ & $\begin{array}{c}\text { oPM.oSM.aSP } \rightarrow o P M . R 1(\neg a S P) \cdot R 2(\neg o S M) \\
=o P M . o S P . a S M(\text { no es una ley) }\end{array}$ \\
\hline
\end{tabular}


Los teoremas de la primera figura y aquellos teoremas de la segunda y de la tercera que se producen a partir de aquéllos requieren una modificación en el algoritmo de decisión.

En efecto, en el caso de los teoremas de la primera hay que «invertir» la regla del descenso. Y en el caso de los teoremas de la segunda y tercera figura que se producen a partir de aquéllos, su prueba producirá un teorema de la primera que habrá de ser transformado en una ley.

Veamos, en primer lugar, los ejemplos y, después, propondremos una modificación del sistema canónico estudiado hasta aquí.

Cuadro 6: Los teoremas de la 1. ${ }^{\mathrm{a}} \mathrm{y}$ los fundados en ellos

\begin{tabular}{|c|c|c|}
\hline Modo problema & Esquema de decisión & Producción \\
\hline$a M P . a S M . i S P$ & $P_{1} . P_{2} . C \rightarrow P_{1} . P_{2} . I D_{i}(C)$ & $\begin{array}{c}a M P . a S M . i S P \rightarrow a M P . a S M . I D 1(i S P) \\
=a M P . a S M . a S P(\text { barbara })\end{array}$ \\
\hline eMP.aSM.oSP & $P_{1} . P_{2} . C \rightarrow P_{1} . P_{2} . I D_{i}(C)$ & $\begin{array}{c}e M P . a S M . o S P \rightarrow e M P . a S M . I D 2(o S P) \\
=e M P . a S M . e S P \text { (celarent) }\end{array}$ \\
\hline aPM.eSM.oSP & $P_{1} \cdot P_{2} \cdot C \rightarrow P_{1} \cdot R_{i} \cdot(\mathrm{J} C) \cdot R_{i}\left(\mathrm{~J} P_{2}\right)$ & aPM.eSM.oSP $\rightarrow a P M \cdot R 2(\neg o S P) \cdot R 3(\neg e S M)=$ \\
\hline$=a P M \cdot a S P . i S M$ & $P_{1} \cdot P_{2} . C \rightarrow P_{1} . P_{2} . I D_{i}(C)$ & $\begin{array}{c}a P M . a S P . i S M \rightarrow a P M . a S . I D 1(i S M) \\
\quad=a P M . a S \cdot a S M(\text { barbara })\end{array}$ \\
\hline ePM.aSM.oSP & $P_{1} \cdot P_{2} \cdot C \rightarrow P_{1} \cdot R_{i}(\neg C) \cdot R_{i}\left(\neg P_{2}\right)$ & $e P M \cdot a S M . o S P \rightarrow e P M \cdot R 2(\neg o S P) \cdot R 1(\neg a S M)=$ \\
\hline$=e P M \cdot a S P . o S M$ & $P_{1} \cdot P_{2} . C \rightarrow P_{1} . P_{2} . I D_{i}(C)$ & $\begin{array}{c}e P M . a S P . o S M \rightarrow e P M . a S P . I D 2(o S M) \\
=e P M . a S P . e S M(\text { celarent })\end{array}$ \\
\hline eMP.aMS.oSP & $P_{1} \cdot P_{2} \cdot C \rightarrow R_{i}(\neg C) \cdot P_{2} \cdot R_{i}\left(\neg P_{1}\right)$ & $e M P . a M S . o S \rightarrow R 2(\neg o S P) \cdot a M S \cdot R 3(\neg e M P)=$ \\
\hline$=a S P \cdot a M S \cdot i M P$ & $P_{1} \cdot P_{2} . C \rightarrow P_{1} . P_{2} . I D_{i}(C)$ & $\begin{array}{c}\text { aSP.aMS.iMP } \rightarrow a S P . a M S . I D 1(i M P) \\
=a S P . a M S . a M P(\text { barbara })\end{array}$ \\
\hline$a M P . a M S . i S P$ & $P_{1} \cdot P_{2} \cdot C \rightarrow R_{i}(\neg C) \cdot P_{2} \cdot R_{i}\left(\neg P_{1}\right)$ & $a M P . a M S . i S P \rightarrow R 4(\neg i S P) \cdot a M S . R 1(\neg a M P)=$ \\
\hline$=e S P . a M S . o M P$ & $P_{1} \cdot P_{2} \cdot C \rightarrow P_{1} \cdot P_{2} \cdot I D_{i}(C)$ & $\begin{array}{c}\text { eSP.aMS.oMP } ® e S P . a M S . I D 2(o M P) \\
=e S P . a M S . e M P(\text { celarent })\end{array}$ \\
\hline
\end{tabular}

Hay que modificar el sistema canónico propuesto más arriba cambiando el apartado IV. Es decir, los esquemas de decisión propuestos han de ser sustituidos por una única regla de decisión, la siguiente:

1. Regla de Decisión (donde $P_{1}$ está por la primera premisa, $P_{2}$ por la segunda y $C$ por la conclusión del modo problema):

$P_{1} . P_{2} \cdot C$ es un modo legítimo si y sólo si

(a) $P_{l} . P_{2} \cdot C \rightarrow P_{l} \cdot R_{i}(\neg C) \cdot R_{i}\left(\neg P_{2}\right)=$ un esquema de axioma o

(b) $P_{1} \cdot P_{2} \cdot C \rightarrow R_{i}(\neg C) \cdot P_{2} \cdot R_{i}\left(\neg P_{1}\right)=$ un esquema de axioma o

(c) $P_{l} . P_{2} . C \rightarrow P_{1} . R_{i}(\neg C) . I D_{i}\left(R_{i}\left(\neg P_{2}\right)\right)=$ un esquema de axioma o

(d) $P_{1} \cdot P_{2} \cdot C \rightarrow R_{i}(\neg C) \cdot P_{2} \cdot I D_{i}\left(R_{i}\left(\neg P_{1}\right)\right)=$ un esquema de axioma. 


\section{CONCLusiones}

Regressus es, a nuestro juicio, un sistema de cálculo para la Silogística eminentemente algorítmico. Esto no implica que rechacemos la interpretación de Couturat. Simplemente creemos que la originalidad de Leibniz consiste en proponer un sistema algorítmico de producción de los modos legítimos que no son de la primera figura. Es lo que hemos probado en la sección 4.

Este sistema puede ser modificado para convertirlo en un método de decisión para la Silogística con tres figuras. Es lo que hemos hecho en la sección 5. Aunque esta idea no sea imputable directamente a Leibniz, sí creemos que está basada en la inspiración general leibniziana de automatizar los procedimientos lógicos.

Antonio Benítez López es Profesor Titular en el Departamento de Lógica y Filosofía de la Ciencia. Facultad de Filosofía. Universidad Compluyense de Madrid. Ciudad Universitaria s/n. 28040 Madrid.

Publicaciones recientes:

(2009): Lógica Bachillera. Madrid. Escolar y Mayo Editores S.L. 150 páginas.

(2009): Apuntes de Historia y Filosofía de la Lógica. Madrid: Cersa. 320 páginas.

Líneas de investigación:

Lógica, Inteligencia Artificial

Correo electrónico: abenitez@filos.ucm.es

José María Benítez Escario es estudiante de doctorado y Becario Complutense predoctoral en el Departamento de Lógica y Filosofía de la Ciencia de la Universidad Complutense de Madrid.

Publicaciones recientes:

(2008): «Optimización de Redes Ad Hoc mediante el algoritmo Ant Colony Optimization», Proceedings of URSI-XXIII, Simposium Nacional de la Union Cientifica Internacional de Radio

(2009): «Autonomous Ship Manoeuvring Planning based on the Ant Colony Optimization Algorithm», 8th IFAC Conference on Manoeuvring and Control of Marine Craft / MCMCO9.

Línea de investigación:

Sistemas artificiales bio-inspirados

Correo electrónico: josemaria.benitez@gmail.com 